\title{
Time-of-Flight Mass Spectrometry of Highly Ordered Carbyne
}

\author{
Yuri Evgenevich Prazdnikov \\ Faculty of Physics, Moscow State University, Moscow, Russia \\ Email: YuriPrazdnikov@yandex.ru
}

Received June 25, 2012; revised July 27, 2012; accepted August 5, 2012

\begin{abstract}
Matrix-assisted laser desorption/ionization (MALDI) time-of-flight mass spectrometry was applied to a non-conventional object - highly ordered carbyne films. Mass spectra of both positive and negative ions were recorded in all tests. The spectra had a rather high number of hydrogen atom peaks and specific features indicative of hydrogen acting as a requisite stabilizer in the crystalline carbyne structure. A new model based on interchain dihydrogen bonds was proposed.
\end{abstract}

Keywords: Carbyne; MALDI; Dihydrogen Bonds

\section{Introduction}

There are numerous modifications of carbyne or linearchain carbon, which is a less understood allotropic $\mathrm{sp}^{1}$ hybridized carbon form [1-4]. Both individual molecules and quasi crystals were obtained experimentally. For the latter, a degree of order can vary from amorphous where a mixture of $\mathrm{sp}^{1}, \mathrm{sp}^{2}$ and $\mathrm{sp}^{3}$ phases is present to a clearly one-dimensional $\mathrm{sp}^{1}$ with the ideal hexagonal packing [2]. Exactly this highly ordered form, i.e. carbyne grown in the form of films from carbon plasma by the ionic stimulation of the surface, is in the focus of this research. The films (at least up to the $1000 \AA$ thickness) are a two-dimensional ordered set of parallel linear carbon chains with a normal orientation to the substrate surface. A distance between the hexagonally packed chains is $5 \AA$ according to diffraction data.

Despite valuable results of some experimental researches [5,6], many scientists have been so far skeptical about the very fact of the highly ordered carbyne existence. A low interest of researchers to this crystalline form is also reasoned by the current unavailability of its recognized consistent model. There are ideas on the polymorphism where a carbine is regarded as an ordered set of carbon macromolecules with kinks, however no shared vision exists with respect to how the macromolecules are ordered and what their topology and interchain distance are. Simulation based on the perturbation theory for different variants of carbynoid systems evidences to their low thermodynamic stability [7]. Pure carbon chain ends have extremely high reactivity. It is believed that all chains in the prepared tangible carbyne end with chemi- cal bonds with hydrogen or other elements, and models have shown their higher stability. Of note is that all stateof-the-art approaches to carbine simulation have a common disadvantage - they compute systems with pregiven spatial coordinates of atoms or with a not broad range of their variance. For instance in $[7,8]$, simulation of relaxation of perturbed atoms of one carbon chain is performed and then minimal free energy of the assembly is computed. Another approach is comprised of simulation of the carbyne generation process such as relaxation of graphite with bonds broken in a certain order [7]. Interestingly is that this technique for the preparation of the highly ordered phase is absolutely different and graphite is known to be a source of amorphous films. Modeling of a highly ordered carbyne growth process would require too many variations of coordinates of a large number of atoms as well as setting of initial velocities, which is presently unfeasible due to limited computational resources. It has been most recently demonstrated that the perturbation theory methods are inapplicable if the effective fine-structure constant achieves a certain critical value [9]. This situation pertains to graphene, what urges the development of a new theoretical approach for computing its electron properties. The value of the constant appeared dependent on the environment. Its value for carbyne has been unknown so far. Nevertheless carbyne, like graphene, is a lower-dimensionality medium where the applicability of any simulation procedure needs an explicit substantiation. Neither of the available carbyne crystal simulations has been clearly substantiated for the time being, so there are grounds for questioning their 
correctness. Anyhow they are worthy of more detailed consideration.

Modeling results for individual carbyne molecules stabilized by bonds with hydrogen not only at the ends but also in intermediates nodes-carbon chain kinks, were communicated in [10]. Such chains, as proposed by the authors, have higher stability and, assumingly, the highly ordered carbyne form consists of exactly these macromolecules with protonated kinks. A linear chain comprised of 6 carbon atoms and terminated with kinks from both ends proved the most energy-favorable. The chemical formula of such carbyne is $-\left[\mathrm{C}_{6} \mathrm{H}_{2}\right]$ - and the hydrogen atoms are located in the same plane as kinked chains. The assumptions on the stabilizing role of impurity atoms, and not just of hydrogen, acting as kink formation sites [3] were put forward even earlier when such atoms had been found in carbyne. Currently it has not been proven experimentally that any element in carbyne is an integral part to its structure rather than an impurity. At present, as we suggest hydrogen to be an integral and stabilizing part of the crystalline carbyne structure, the best-recognized Heimann model of today [3] needs to be supplemented. In the Heimann model, kinks separate identical layers shifted relative to each other whereas the kink plane orientation is random and is in no way tied up to the hexagonal symmetry of an individual layer. The addition of two hydrogen atoms to each kink necessitates accounting for the interaction of these atoms with neighboring chains. The interaction should impose conditions on the orientation of the chain planes.

Some experiments demonstrated a high orderliness of carbyne carbon chains. These are primarily electron diffraction and X-ray microanalysis data that showed an ideal hexagonal packing [5]. The indirect evidence of linear chains conservation was tunnel transparency of rather thick carbyne films as well as excellent repeatability of experiments on the charge carrier injection from carbyne films and injection current independence from the carbyne film thickness (from 20 to $1000 \AA$ ) [11]. Thus, if kinks do exist along the chains, they should not lead to a loss of long-range order of the chain. It is possible in the instance of kinks alternating the direction of the parallel shift of all chains in the chain plane, as shown in Figure 1. The authors [8] propose a similar modification of the Heimann model with the difference that chains are supposed to be not linear but a little curved in the chain's plane.

As seen from Figure 1, some hydrogen atoms (located in the chain plane as required by chemical bonds in the kink area) of neighboring chains sit closer to each other than the chains themselves. Theoretically here should occur an interaction of polarized chains (hydrogen as an electron donor is positively charged and carbon-negatively), whose three-dimensional analog is found in water mo-

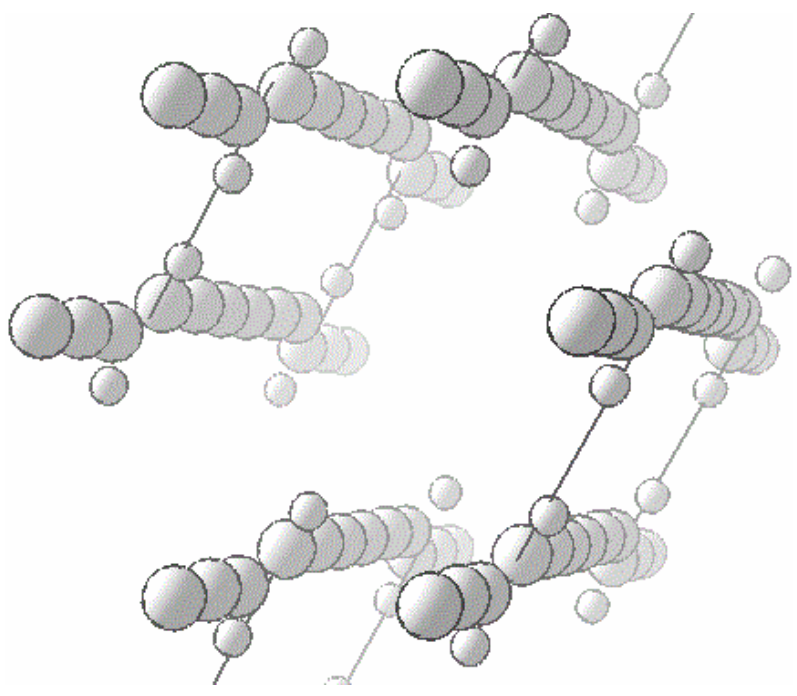

Figure 1. A 3D-Heimann model of the carbyne crystal supplemented with hydrogen (small balls) in the kink area. Minimal distances between neighboring chains are shown by hydrogen bonding sections.

lecules. The position shown in Figure 1 should be unstable: positively charged hydrogen atoms sit most closely and here mutual repulsion occurs. The rotational degree of freedom prevents the chains from finding a stable position because each chain is symmetrically surrounded by three neighboring chains and the chains have flat topology. Even if to assume that a certain stable configuration is in place, the bonds emerging between individual chain pairs would be definitely insufficient to hold chains with lengths of roughly 1000 atoms. A weak point of the model with pure chemical bonds in the kink area is its topological inconsistency due to the impossibility of triple symmetry. Forces that would have trilateral symmetry corresponding to the three nearest neighboring chains should exist between the chains. It is known from the experiments that highly ordered carbyne films as low as $20 \AA$ thick have the same injection properties as films with the thickness of $1000 \AA$ [11]. The particularity of the chain build-up technology is that the chains several atoms long precipitate in one pulse. Apparently, the chains form a hexagonally ordered lattice at the very first precipitation pulse. But how are they ordered? What forces hold them in a vertical position?

To answer these questions we should assume that dihydrogen bonds exist between the chains. A detailed study [12] on the intermolecular dihydrogen bonding allowed establishing that the $\mathrm{H}-\mathrm{H}$ distance (by X-ray analysis data) is within the $1.7-1.9 \AA$ range. This value is less than the sum of van der Waals radii of two hydrogen atoms $(2.4 \AA)$, which confirms the existence of this unusual bonding. One may say that the dihydrogen bonding is, to some extent, an exotic phenomenon to be found in hydride chemistry and has no analogs in organic 
chemistry. Anyhow recently the notion of "dihydrogen bonding" has expanded to cover diverse transformations with the participation of a wide scope of chemical elements. We suppose an ordered carbyne to be a quasi crystal that exists owing to dihydrogen bonds (Figure 2). The distance of $5 \AA$, at which chains in carbyne are spaced apart from each other, is typical for such bonds. The dihydrogen bonding existence is conditioned by available opposite charges on both sides on the hydrogen bridge. This charge distribution is typologically uncontroversial with the hexagonal order (on the right in Figure 2). Assumptions concerning the existence of some "built-in" field were put forward before with a view to possibly explain the abnormal injection emission capability [11]. Its source could be spin-charged solitons similar to those in conjugated polymers [13]. In such model, high conductivity of highly ordered carbyne may have the same nature as doped copolymers. Carbyne chains should demonstrate the semiconductor properties due to dimerization of carbon atoms, yet the experiments show metal-level conductivity.

\section{Results and Discussion}

To verify the proposed model we conducted experiments using the matrix-assisted laser desorption/ionization (MALDI) mass spectrometry technique that has quite recently become a tool for researching carbon materials. Desorption is conventionally performed with the use of a special matrix that absorbs well laser pulses. It acts as a carrier for the substance under study and evaporates with it from the substrate. We used a carbyne film (thickness-approximately $100 \AA$ ) as such matrix, stemming from an assumption that the film that thick should absorb photon energy and evaporate as fragments. MALDI mass spectra were registered on a reflector time-of-flight mass spectrometer Bruker AutoFlex II (Germany) fitted with a $337 \mathrm{~nm}$ wavelength and $1 \mathrm{~ns}$ pulse nitrogen laser. Fragmentation and ionization mechanisms are rather sophisticated and can proceed via many diverse scenarios, some of which take place as early as during the flight to an analyzer. We assumed that transformations in the fragments already after evaporation made roughly equal noise contributions in the overall mass range without any particularities. The most pronounced specific features of the spectra were assigned to the specifics of the highly ordered carbyne structure and primary fragmentation.

The mass spectra, of both positive and negative ions, were obtained for carbyne films (Figures 3 and 4). By the peaks period, the spectra almost fully correspond to the $\mathrm{C}_{\mathrm{n}}$ carbon chain fragments with maximal intensity at $\mathrm{n}=4$ and $\mathrm{n}=6$ for positive and negative ions, accordingly. The most characteristic detail here is a significant number of hydrogen satellite peaks $\mathrm{C}_{\mathrm{n}} \mathrm{H}_{\mathrm{m}}$. A mismatch between the values and the theory (e.g. for $\mathrm{C}_{2} \mathrm{~m} / \mathrm{z}=22.8$ instead of 24) is especially noticeable in the initial region of the spectrum, although the instrument had not been calibrated in the range of so small masses. However, the match is not bad already for $C_{10}(119.9)$ - this point was taken as a basis for superimposing the theoretical scale $\mathrm{C}_{\mathrm{n}}$ on the axis $\mathrm{m} / \mathrm{z}$ in Figure 4.

The positive spectrum has a worse match with theoretical $C_{n}$ (a larger period and more satellite peaks) than the negative one. We explain this discrepancy by the fact that the former is comprised of the fragments, which better retained the structure of bonds with hydrogen, and the

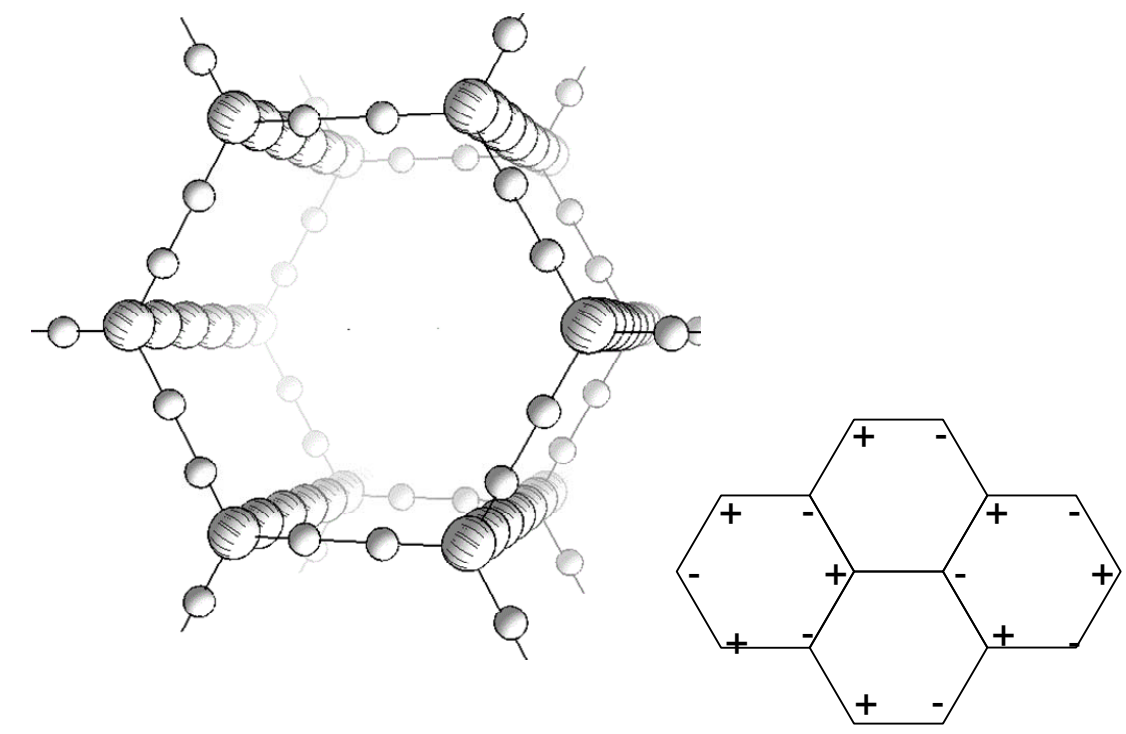

Figure 2. A new 3D-model of the carbyne crystal based on dihydrogen interchain bonds. Interchain bonds-through each two hydrogen atoms (small balls) - build a plane that links carbon chains. The assumed charge distribution in the plane (top view) is shown on the right. 


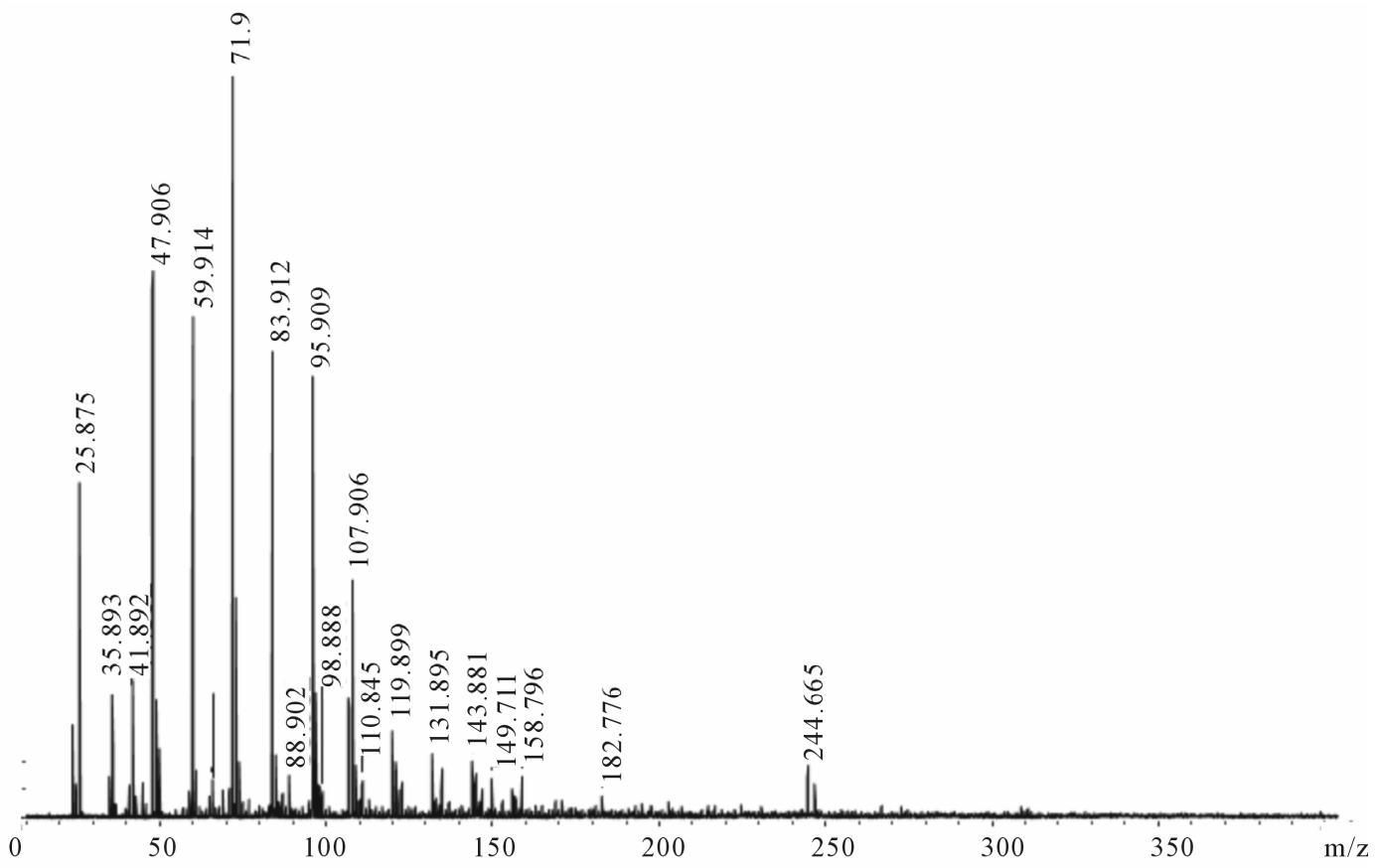

(a)

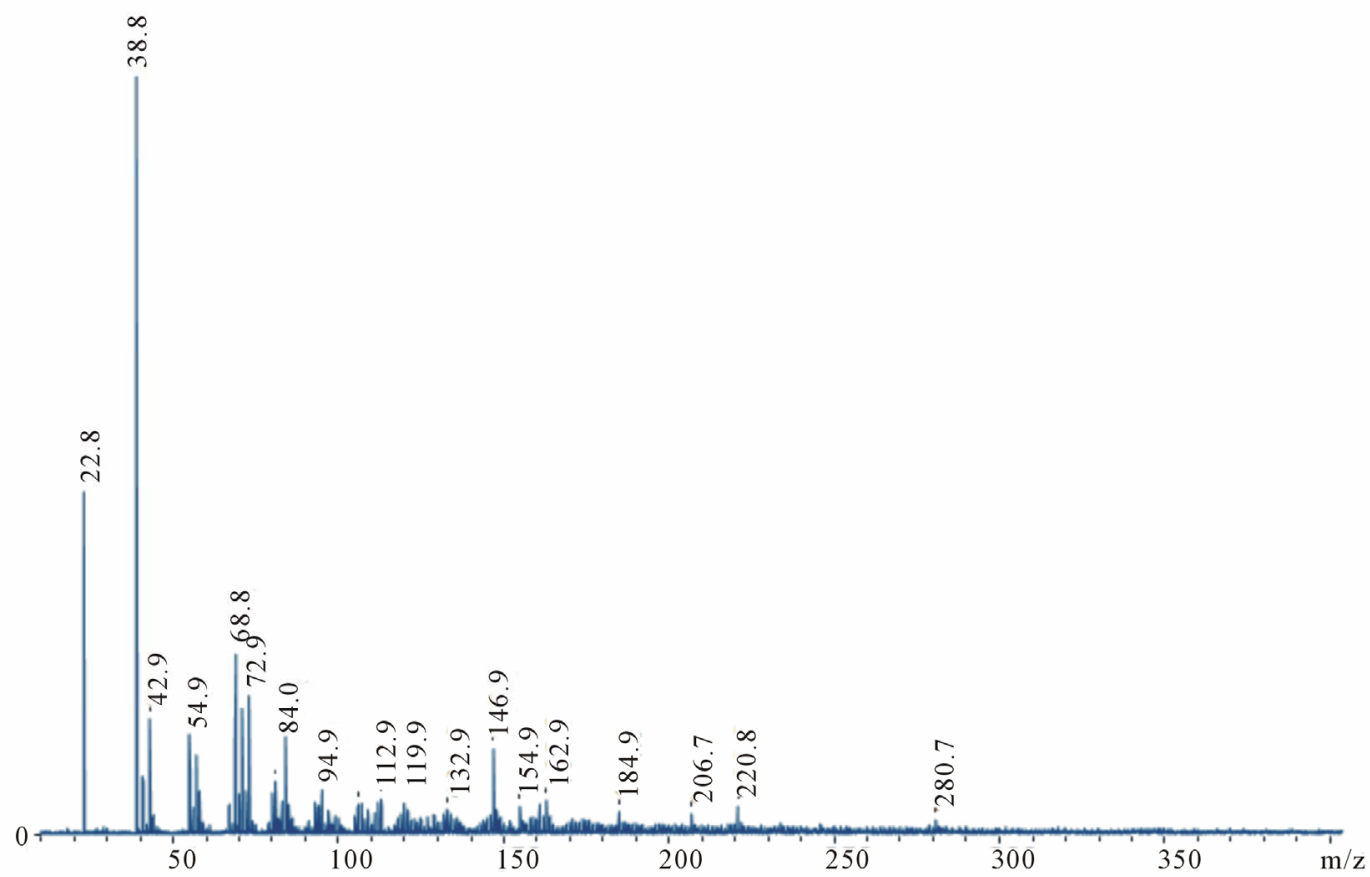

(b)

Figure 3. The carbyne mass spectra in the $\mathrm{m} / \mathrm{z} 0$ - 400 range. (a) Positive ions; (b) Negative ions.

latter is formed of actually pure carbon chains. It is easy to see on the extreme right peaks: Whereas in the negative spectrum the peak $\mathrm{m} / \mathrm{z}=245\left(\mathrm{C}_{20}\right)$ is clearly displayed, the positive spectrum shows merely the blurred background of its hydrogen satellites with a small maximum $\mathrm{m} / \mathrm{z}=280$. Evidently, there are two types of bonds with hydrogen atoms that differ greatly in energy: In negative ions the peak $\mathrm{C}_{20}$ has a hydrogen satellite $\mathrm{C}_{20} \mathrm{H}_{2}$ with comparable intensity. This can be explained by two terminal chemical bonds with hydrogen, which are stronger than side sorption bonds with hydrogen along the chain. The absence of the peak $\mathrm{C}_{20} \mathrm{H}$ may be indicative of instability of the chain that has terminal hydrogen only on one side. A reason for retaining side hydrogen 


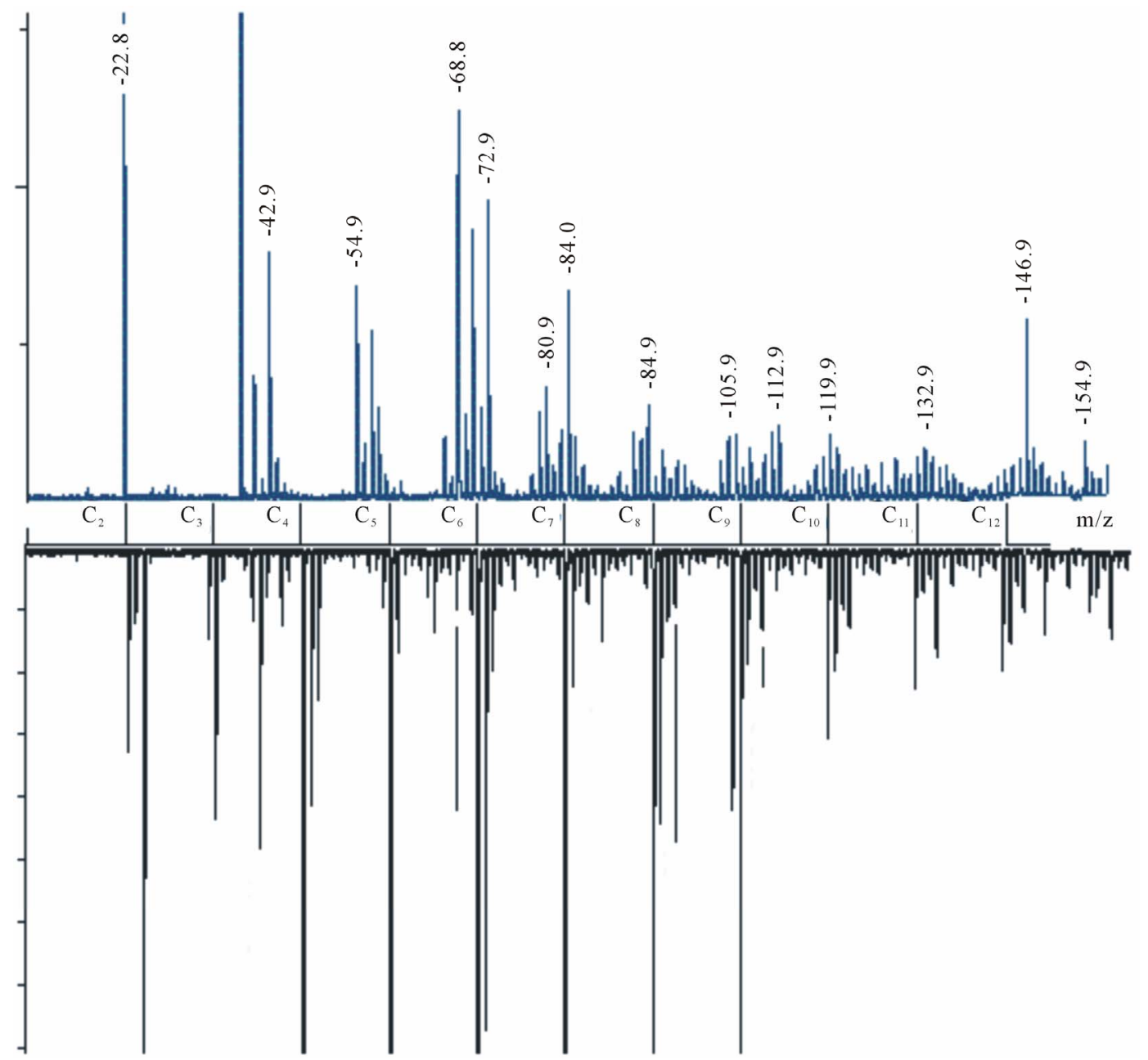

Figure 4. The most important fragments of the carbine mass spectra are zoomed. The top figure shows positive ions, the bottom figure-negative ions (specular reflected relative to the horizontal axis). Relative intensity-by the vertical axis. Theoretical values $\mathbf{m} / \mathbf{z}$ for pure carbon chains $C_{n}$ are given by the horizontal axis.

atoms in positive ions is likely to be related to the suggested electron density distribution in the initial carbyne structure (Figure 2). In the course of the fragmentation, a break of the dihydrogen bond occurs near $\mathrm{C}^{-}$and a pure carbon fragment and a fragment with adsorbed neutral hydrogen molecules are formed near $\mathrm{C}^{+}$. The positive spectrum of peaks $\mathrm{C}_{4} \mathrm{H}_{\mathrm{m}}$ and $\mathrm{C}_{6} \mathrm{H}_{\mathrm{m}}$ is clearly marked with a prevailing even character $\mathrm{m}$. For the fragments $\mathrm{C}_{6} \mathrm{H}_{\mathrm{m}}$, the peak of the pure chain $\mathrm{m}=0$ and all three hydrogen peaks that, assumingly, correspond to ions $\mathrm{C}_{6} \mathrm{H}_{2}, \mathrm{C}_{6} \mathrm{H}_{4}$ and $\mathrm{C}_{6} \mathrm{H}_{6}$ are distinctly seen, whereas the pure carbon fragments are much less in number. It is the most interesting peculiarity, which corresponds to the desorption of the carbon chain fragment with adjacent interchain dihydrogen bonds (Figure 5).

If we try to explain this series of peaks in terms of chemical bonds, $\mathrm{C}_{6} \mathrm{H}_{6}$ could be a ring structure of benzene. In this case it is however unclear why hydrogen atoms detach from this molecule pairwise and why the same pairwise pattern persists in the $\mathrm{C}_{4}$ series. These peculiarities are even less understandable within the extended Heimann model (Figure 1). Our model allows a better understanding of these specific features. The interchain dihydrogen bond break occurs by the $\mathrm{C}-\mathrm{H}$ bond rather than by $\mathrm{H}-\mathrm{H}$. To this end, we can observe the pairwise hydrogen atoms in the recorded spectrum. The number of $\mathrm{H}_{2}$-peaks is 3 (Figure 5), which is in good agreement with our model since each chain has 3 neighboring atoms (Figure 2). Of note is that the number of adsorbed hydrogen molecules is limited by three and they all are remains of dihydrogen bonds of one interchain plane. Should carbyne be a strictly periodic crystal, e.g. with a 6 atoms period, peaks corresponding to bonds from two interchain planes could be present in the $\mathrm{C}_{12} \mathrm{H}_{n}$ spectrum. The spectra evidence to the absence of such periodicity, what is likely to relate both to the topological differences between neighboring layers in the carbyne film and to the charge distribution. As seen from Figure 2 (on the right), 


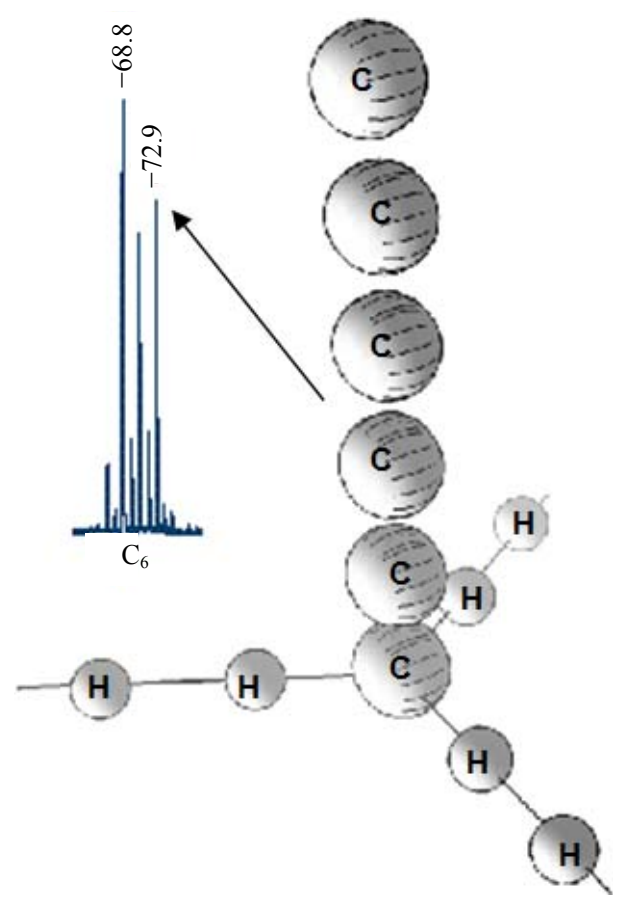

Figure 5. One of the highly ordered carbyne crystal fragments, assumingly, recorded on the mass spectrum of positive ions as a peak $\mathrm{m} / \mathrm{z}=72.9$ in a series of peaks $C_{6}$. The pairwise character of the hydrogen atoms fragmentation is clearly seen.

two different charge distributions can exist in the plane of interchain dihydrogen bonds that correspond to the charge wave along the chain.

An interesting feature here is a lack of pronounced "magic" numbers in the $\mathrm{C}_{\mathrm{n}}$ spectra, which could be indicative of the alteration period of transversal dihydrogen bonds in carbyne. In the strict sense, it does not mean that the period is not constant as the very highly ordered carbyne fragmentation and ionization processes are sophisticated and unexplored. It is more probable that carbyne does not have this constant period regardless of earlier suppositions that carbyne singularly consists of linear carbon fragments with $n=4$ or $n=6[5,10]$. For a constrained polyyne chain terminated by bonds with hydrogen from both ends theoretically was obtained a formula of "magic" lengths of carbon atoms per chain $\mathrm{N}=$ $4 n+7$ (n-natural), as follows from a possibility of the existence of spin-charged solitons in such chains [14]. Enhanced stability of carbon chains with $\mathrm{N}$ equal to 11 , 15,19 and 23 was ascertained by TOF mass spectrometry of the graphite laser-evaporated products [15]. Another sequence of "magic" thicknesses of overlapping highly ordered carbyne layers was marked by the authors [13]: 10, 85, 225 and $1150 \AA$, which corresponded to 8, 68,180 and 920 atoms approximately. The emergence of the layers, as the film grows, is expressed through conductivity jumps measured transversely to the chains. Hy- drogen was not specially introduced during the ongoing growth of highly ordered carbyne films. As we suppose, it is needed for transverse stability. Therefore the emergence of the exponentially growing spectrum of successive layer thicknesses could be explained by a limited hydrogen stock in the chamber during the growth. It should be pinpointed that the spectrum pertains exactly to the highly ordered form studied here, whereas the periodic spectrum of "magic" thicknesses $N=4 n+7$ relates to free chains formed in the carbon plasma after the graphite laser ablation. The attained mass spectra testify to rather low stability of free long chains in vacuumeach fixed chain has no more than 30 atoms. Hence it is possible to view only the whole fragments of the thinnest "magic" thickness from the sequence.

A content of other atoms, apart from hydrogen and carbon, in the spectra is minor. This is consistent with unpublished experimental results for carbine film [13] whereto doping atoms such as sulfur and nitrogen were added while growing. The measurements of cross-chain resistance showed that merely pure films gave clear repetitive current-voltage characteristics. According to the band model all dopants act as traps located in the bandgap, viz. are not part to the crystal periodic structure. So we may conclude that singularly hydrogen with its unique ability to build dihydrogen bonds is capable of self-organizing with carbon to an ideal structure of the carbyne crystal.

\section{REFERENCES}

[1] A. M. Sladkov, V. I. Kasatochkin, V. V. Korshak, et al., "Inventor's Certificate No. 107 (December 7, 1971)," Bulletin Izobrebereteny, No. 6, 1972, p. 3.

[2] Yu. P. Kudryavtsev, M. B. Guseva and V.G. Babaev, "Oriented Carbyne Layers," Carbon, Vol. 30, No. 2, 1992, pp. 213-221. doi:10.1016/0008-6223(92)90082-8

[3] R. B. Heimann, S. E. Evsyukov and L. Kavan, "Carbyne and Carbynoid Structures," Kluwer, Dordrecht, 1999. doi:10.1007/978-94-011-4742-2

[4] C. S. Casari, et al. "Chemical and Thermal Stability of Carbyne-Like Structures in Cluster-Assembled Carbon Films," Physical Review B, Vol. 69, 2004, Article ID: 075422. doi:10.1103/PhysRevB.69.075422

[5] V. Babaev, M. Guseva, V. Khvostov, N. Novikov and P. Flood, "Carbon Material with Highly Ordered LinearChain Structure, in 'POLYYNES-Synthesis, Properties, Applications'," CRC Press, Boca Raton, 2005, pp. 219252.

[6] Yu. Prazdnikov, "Prospects of Carbyne Applications in Microelectronics," Journal of Modern Physics, Vol. 2, No. 8, 2011, pp. 845-848. doi:10.4236/jmp.2011.28100

[7] W. Luo and W. Windl, "First Principles Study of Structure and Stability of Carbynes," Carbon, Vol. 47, No. 2, 2009, pp. 367-383. doi:10.1016/j.carbon.2008.10.017 
[8] Yu. G. Korobov and D. I. Bazhanov, "Ab initio Research on the Structure of Linear-Chain Carbon Oriented Films," JETP Letters, Vol. 95, No. 9, 2012, p. 524.

[9] J. E. Drut and T. A. Lähde, "Is Graphene in Vacuum an Insulator?" Physical Review Letters, Vol. 102, 2009, Article ID: 026802. doi:10.1103/PhysRevLett.102.026802

[10] J. G. Korobova and D. I. Bazhanov, "Effect of Hydrogen on the Formation of the Atomic Structure of Linear Carbon Chains: An ab initio Approach," Journal of Experimental and Theoretical Physics Letters, Vol. 93, No. 11, 2011, pp. 652-656. doi:10.1134/S0021364011110063

[11] Yu. E. Prazdnikov, A. D. Bozhko and N. D. Novikov, "Energetic Barrier Reduction at the Carbyne Film Interface," Journal of Russian Laser Research, Vol. 26, No. 1, 2005, pp. 55-65. doi:10.1007/s10946-005-0006-4

[12] N. V. Belkova, L. M. Epstein and E. S. Shubina, "Dihydrogen Bonding, Proton Transfer and Beyond: What We
Can Learn from Kinetics and Thermodynamics," (Microreview), European Journal of Inorganic Chemistry, Vol. 2010, No. 23, 2010, pp. 3555-3565. doi:10.1002/ejic.201000546

[13] Yu. E. Prazdnikov, L. S. Lepnev, A. D. Bozhko and N. D. Novikov, "Conductance Spectra of Carbyne Transverse to Carbon Chains. Is It Related to the Soliton Lattice?" Journal of Russian Laser Research, 2005, Vol. 26, No. 3, pp. 245-251. doi:10.1007/s10946-005-0017-1

[14] M. J. Rice, S. R. Philpot, A. R. Bishop and D. K. Campbell, "Solitons, Polarons, and Phonons in the Infinite Polyyne Chain," Physical Review B, Vol. 34, No. 6, 1986, p. 4139. doi:10.1103/PhysRevB.34.4139

[15] S. W. McElvany, M. M. Ross and J. H. Callahan, "Characterization of Fullerenes by Mass Spectrometry," Accounts of Chemical Research, Vol. 2, No. 3, 1992, pp. 162-168. doi:10.1021/ar00015a010 